\title{
Viviendo con teléfono y pareja. Usos del celular en entornos de alta conectividad
}

\author{
Living with a phone and a partner. Cell phone \\ uses in high connectivity environments ${ }^{*}$
}

Abimee Viridiana González Rodríguez**

Docente en la Universidad Estatal de Sonora ORCID: https://orcid.org/oooo-000I-8I2I-3822

Lorenia Velázquez Contreras ${ }^{\star * *}$

Profesora-investigadora de El Colegio de Sonora ORCID: https://orcid.org/0000-0002-1367-I00I

Gabriela Grijalva Monteverde**** Profesora-investigadora de El Colegio de Sonora ORCID: https://orcid.org/o0oo-000I-9010-3254

ISSN-OI85-4259; e-ISSN: 2007-9I76

\section{Resumen}

DOI: http://dx+doi.org/IO.28928/ri/892020/aot3/gonzalezav/velazquezl/grijalvag

Los indicadores sobre el uso generalizado de tecnologías de la información y la comunicación (TIC) limitan la identificación de brechas entre los distintos grupos de población. Este trabajo tiene como objetivo contribuir a la exploración de las relaciones de la telefonía móvil con el desempeño de los roles de género en México, mediante una aproximación cuantitativa para identificar formas e intensidades en que personas que viven con parejas del sexo opuesto se apropian y utilizan el teléfono celular. Se generó información primaria mediante la aplicación de encuesta en un medio urbano del norte de México con alta conectividad (la ciudad de Hermosillo, Sonora). Los resultados muestran la persistencia de una de las formas de exclusión digital de las mujeres, así como redefiniciones de las jerarquías de género al identificar diferencias en acceso y uso de TIC — particularmente del teléfono celular- no identificables en las estadísticas disponibles en México.

Palabras clave: tecnologías de la información y la comunicación, brecha digital de género, Sonora, México.

\footnotetext{
Abstract

Indicators on the widespread use of information and communication technologies (ICT) limit the identification of gaps between different population groups. This exploratory work aims to find the relationships of mobile telephony with the performance of gender roles in Mexico, providing a quantitative approach to identify ways and intensities in which individuals living in heterosexual couple use and appropriate the cell phone. Primary information was generated by applying a survey in northern Mexico in a high-connectivity urban environment (the city of Hermosillo, Sonora). The results show the persistence of one of the forms of digital exclusion of women as well as redefinitions of gender hierarchies when identifying differences in access and use of ICT - particularly cell phone - not identifiable in data available in Mexico.
}

Keywordsः ICT, digital gender gap, Sonora, México

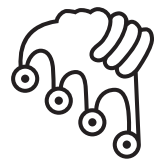

\section{IZTAPALAPA}

Agua sobre lajas

* Agradecemos las observaciones que realizaron dos revisores anónimos, que nos permitieron mejorar este trabajo.

**ahimee.gonzalez@ues.mx

***1velaz@colson.edu.mx

****ggrijal@colson.edu.mx 


\section{INTRODUCCIÓN}

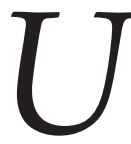

na expresión de desigualdad, identificada como limitante para el desarrollo de la llamada sociedad del conocimiento, es la división o brecha digital de género que, de inicio, alude a la diferencia en las proporciones en que hombres y mujeres acceden a las tecnologías de la información y la comunicación (TIC). Esta definición se ha ampliado con argumentos de autoras y autores con diversos compromisos teóricos e institucionales (Bimber, 2000; Servon y Pinkett, 2006; Castells et al., 2007; Mariscal, 2009; Hilbert, 20II; uit/unesco, 20I3), donde prevalecen aquellos aportes que desde una perspectiva de género señalan que la brecha también está en la forma como se aprovechan las tecnologías por los distintos usuarios y que consideran el género como un factor primordial para el análisis (origen) de las diferencias (Castaño, 2008; Gil-Juárez et al., 20II; Lasén, 2014; Vega, 2016).

Las Tic facilitan el manejo e intercambio de información, así como la comunicación entre seres humanos, sistemas electrónicos y al interior de estos (Huyer y Hafkin, 2006). Incluyen radio, televisión análoga y digital, teléfono fijo y móvil, computadoras, videojuegos, internet y sus aplicaciones: correo electrónico, redes sociales virtuales, programas accesibles en línea, entre otras.

El teléfono celular es la Tic que mayor difusión obtuvo a partir de su introducción comercial en Europa y Estados Unidos en la década de 1990. Desde entonces, rápidamente pasó de ser un dispositivo de lujo con dimensiones poco portátiles y cuya utilidad de transmisión móvil de voz era poco comprendida, a convertirse en un dispositivo de acceso generalizado en la población, con características de uso cada vez más sofisticadas, como el acceso a internet móvil de alta velocidad.

En México, según estadísticas oficiales, la proporción de hombres y mujeres con acceso a las TIC es tan similar que a primera vista es difícil distinguir una brecha digital de género. El objetivo de este trabajo es contribuir a la exploración de las relaciones de la telefonía móvil con el desempeño de los roles de género en México, aportando una aproximación cuantitativa para identificar las formas e intensidades en que personas que viven en pareja (heterosexual) se apropian y usan el teléfono 
celular, con base en información primaria generada por encuestas en un medio urbano del norte de México con alta conectividad (la ciudad de Hermosillo, Sonora) y la propuesta de análisis de Amparo Lasén (2014). Se asume la existencia de diferencias y condicionantes específicas de género en las maneras en que se accede y se usa la telefonía móvil desde una visión conceptual de la microfísica del poder y la subjetividad según Foucault (1976; 1988) y que Lasén (2014) matiza para explicar las subjetividades de género mediadas por la tecnología. El planteamiento aquí abordado considera el teléfono móvil como un dispositivo de comunicación humana que no puede descontextualizarse de las estructuras sociales en que se utiliza y que, bajo esas consideraciones, participa en la extensión, definición y redefinición de las relaciones y los estereotipos que establecen distintos espacios, valoraciones y tareas para hombres y mujeres en cualquier organización social, conformando una expresión de la brecha digital de género poco abordada en la literatura en México o publicada en español. Lasén y Casado (2014) hacen notar, después de una revisión exhaustiva de la literatura en castellano, la escasez de aproximaciones teóricas, pero sobre todo la insuficiencia de estudios de campo acerca de la comunicación digital y las subjetividades, entre ellas las de género; el presente trabajo busca contribuir en este sentido.

Los datos presentados provienen de una muestra exploratoria de 53 mujeres y hombres adultos, que encabezan sus respectivos hogares y cohabitan con una pareja del sexo opuesto, residentes de Hermosillo, capital del estado de Sonora, considerada como la ciudad de mayor conectividad en México (INEGI, 20I8).

Centrar el interés sobre este grupo permitió obtener información acerca de los usos del teléfono celular que se dan en las relaciones de pareja heterosexual, especialmente para atender los asuntos relacionados con el ámbito familiar, las labores de cuidado y el trabajo extradoméstico. ${ }^{1}$

La primera parte es resultado de la revisión de estudios sobre las desigualdades de género en el acceso y uso de las Tic y de las diferencias observadas en los usos del teléfono celular (Castells et al., 2007; Mariscal, 2009; Johnson, 2013; Jouhki, 2013; Lasén, 2014). Posteriormente se subrayan las aportaciones teóricas de Foucault (1976; 1988) y Bartky (1994) que dan marco a la interpretación sumando la postura de Lasén (2014) que proporcionó un referente metodológico para guiar las preguntas de investigación.

1 Lasén (2014) realiza su estudio desde un enfoque cualitativo con parejas, considerando también aspectos socioeconómicos, laborales y familiares, así como la división de tareas, mediados por la telefonía móvil. 
La segunda parte muestra el diseño metodológico del operativo de campo que generó los datos para el análisis que se presenta en los apartados de resultados y discusión. La última parte contiene las conclusiones de la investigación donde se describen los hallazgos de diferenciación cualitativa entre hombres y mujeres en cuestión de acceso y uso de las Tic y se subraya la necesidad de generar indicadores específicos para hacer visibles las divisiones digitales.

\section{Revisión teórica}

\section{La perspectiva de género y la brecha digital}

En su forma más general, el concepto de brecha digital describe desigualdades mostradas entre personas de diferentes países, regiones o grupos de población en cuanto al acceso y dominio de Tic. Algunos autores apuntan la necesidad de acercarse al concepto desde diferentes posturas para comprender las manifestaciones del problema; plantean que la brecha digital se refiere no solamente al acceso, sino también al uso que se le da a computadoras e internet, los contenidos que se buscan y a cuáles se puede acceder a partir de las oportunidades de educación, empleo, ingresos y alfabetización digital de que se dispone (Bimber, 2000; Servon y Pinkett, 2006; Castaño, 2008; Gil-Juárez et al., 20II; Hilbert, 20II; UIT/UnesCO, 20I3; Alva, 2015; Pujol y Montenegro, 2015; Vega, 2016). Así, la concepción de brecha digital de género no debiera reducirse a un término binario acerca de si hombres y mujeres tienen acceso o no a las TIC, sino que debe ampliarse y matizarse observando las distintas dimensiones del fenómeno marcadas por el género. ${ }^{2}$

Aun cuando la brecha digital es reducida, está presente en las múltiples formas en que las mujeres se alejan de las Tic en el transcurso de su vida educativa y laboral, y se explica porque a través de la socialización de género se aprenden normas, valores y formas diferentes de hacer y decir de acuerdo con el sexo, y esto también

2 De acuerdo con la Conferencia de Naciones Unidas para el Comercio y el Desarrollo (UNCTAD, por sus siglas en inglés), el interés en hacer visibles las desigualdades y otros asuntos relacionados con el género y las Tic tiene que ver no solamente con la igualdad de derechos y el acceso a la información, sino también con la perspectiva de incrementar las oportunidades económicas de las mujeres mediante las Tic. Aunque de forma muy sintética, también en la Agenda 2030 para el Desarrollo Sostenible (ADS-2030) se plantea la posibilidad de empoderamiento de las mujeres a partir de las Tic. 
se traslada a la forma de relacionarse con la tecnología (Gil-Juárez et al., 20II), la cual se percibe socioculturalmente como un ámbito de actuación primordialmente para varones (Wajcman y Jones, 20I2; Wajcman, 2000).

Las autoras españolas Lasén y Casado (20I4) analizan las subjetividades inmersas en medios tecnológicos en consonancia con los estudios constructivistas de la tecnología. Desde la perspectiva de estas autoras, con la tecnología se constituyen vínculos ya sea al generar usos particulares o mediante las resistencias a desplegarla en todas sus funcionalidades.

De esta manera, la aproximación a la tecnología puede explicarse en más de una dimensión, no únicamente en la posesión de la herramienta, sino en cómo se apropia y se incluye en la cotidianidad de las relaciones sociales, en las que se entrecruzan, además de la estructura y subjetividades de género, otras categorías como el nivel socioeconómico, la etnia y factores sociales relacionados con la familia, el trabajo y la educación.

En la construcción social de género, la naturaleza biológica es solamente un fundamento sobre el que se edifican las ideas que otorgan valoraciones desiguales a hombres y mujeres para legitimar o naturalizar las relaciones de dominación en todo el entramado institucional en el que se desenvuelven las personas: en hogares $y$ familias, en la escuela, en el mercado de trabajo, en la Iglesia y el Estado (Bourdieu, 2000). Esta persistencia y ubicuidad convierten el género en "el campo primario dentro del cual o por medio del cual se articula el poder" (Scott, 1996: 292). La perspectiva de género pone de relieve la desigualdad de las relaciones sociales que se construyen alrededor de las diferencias biológicas entre los sexos, donde lo considerado femenino es sistemáticamente desvalorizado frente a lo masculino y las mujeres son situadas históricamente en una posición subordinada (Brito, 2016; De Barbieri, 1993). Así, pese a que las mujeres logren igualdad de acceso a ciertos espacios como la escuela, la tendencia en el plano de lo laboral es que ejerzan actividades menos valoradas, más vinculadas al cuidado, como la enseñanza o los servicios de salud y, además, se vean limitadas en el ascenso a espacios de poder o puestos más altos, como los gerenciales (Bordieu, 2000; Brito, 2016).

Castaño (2008) denomina segunda brecha a la determinación y el análisis de la utilización e intensidad de uso de internet y resalta que los puestos de trabajo, como vía de acceso inicial, delimitan la experiencia y las habilidades en el uso de ordenadores e internet y destaca que el empleo femenino se ubica en posiciones subordinadas o poco relevantes, incluso entre quienes laboran en áreas de informática. También remarca la importancia del contexto familiar —el cual afecta las posibilidades económicas de acceder a computadora y conexión a internet- - cómo 
la presencia de menores de edad en el hogar influye negativamente en el tiempo disponible de las mujeres.

En el contexto mexicano, las condiciones de pobreza, marginación y violencia de género condicionan el uso de las Tic por parte de las mujeres, quienes - aparte de atender cuestiones de trabajo o recreación - utilizan la comunicación por internet para consumo de servicios relacionados con las necesidades de la familia de manera más frecuente que los varones (Vega, 2016).

\section{La brecha digital de género y el caso del teléfono celular}

El teléfono celular es la tecnología de la información que mayor difusión ha tenido desde la última década del siglo xx. Inicialmente, la comunicación móvil fue diseñada y comercializada para adultos - primordialmente varones - en círculos empresariales; sin embargo, la difusión posterior atrajo en gran medida a adolescentes y adultos jóvenes, quienes disponían de tiempo libre, recursos económicos y no tenían líneas telefónicas fijas (Castells et al., 2007).

Desde entonces, el celular se desarrolló y comercializó hasta transformarse en un aparato de acceso generalizado en la población y con características de uso cada vez más sofisticadas como el acceso a internet de alta velocidad. Una de las mayores barreras para su uso en América Latina se ha relacionado con el costo del servicio (Mariscal, 2009); en cuanto al sistema de facturación, para el año de referencia del estudio de Mariscal (2009), solo 7\% utilizaba un sistema de pospago contra 92\% que usaba los sistemas de prepago, porque lo consideran una mejor forma de controlar su presupuesto (Mariscal, 2009; Ruelas, 2014).

Algunos países en desarrollo han aprovechado la facilidad en el acceso para disminuir la brecha digital, utilizándola con fines de desarrollo social, tales como educación y salud a distancia, servicios bancarios y de envío de remesas o acceso a internet inalámbrico.

En cuanto a las brechas presentes en la comunicación móvil, en el estudio de Castells et al. (2007) se distinguen diferencias de adopción en las categorías de edad, género, nivel socioeconómico e identidad étnica; por ejemplo, entre la población de menores ingresos, la telefonía móvil se usa principalmente para estar en contacto y estrechar lazos con la red social y en menor medida para asuntos de trabajo o negocios.

Estos mismos autores discuten las diferentes posturas acerca de si la telefonía móvil es liberadora de las limitaciones de género o tiende a reforzarlas. Señalan que una de las características de apropiación hallada en la mayoría de los países es que 
las mujeres posean un celular motivadas por un sentimiento de seguridad y protección, y que los hombres provean a sus hijas o esposas de un teléfono con el mismo propósito. También advierten que las mujeres tienden mayormente a mantener sus redes sociales a través del teléfono, así como a utilizarlo para coordinar actividades familiares (Castells et al., 2007; Kumar et al., 2017).

Otro aspecto destacado de la telefonía móvil es dotar de ubicuidad a quien lo usa: puede conectarse con el trabajo o los seres queridos casi desde cualquier sitio. Quienes trabajan fuera de casa son accesibles desde la oficina a cualquier hora del día, sea en casa o en medio del tránsito. Los hijos pueden ser tele-cuidados mediante llamadas constantes para verificar que todo esté bien; las amas de casa pueden ser requeridas y localizadas por el resto de la familia, aunque no estén en la casa, de modo que las desigualdades de género persistan a través del teléfono móvil: los hombres pueden estar ampliando su actuación pública y trayéndola a su vida personal, en tanto que las mujeres pueden estar ampliando su presencia en el ámbito público sin disminuir sus responsabilidades en el privado (Castells et al., 2007).

\section{Foucault y la subjetivación a través del teléfono celular}

La tecnología celular y las implicaciones de sus características de movilidad y ubicuidad para las relaciones sociales de género pueden abordarse a través de Foucault (1976; 1988), quien analiza las relaciones de poder y los procesos de subjetivación, esto es, la constitución de sujetos. Para el autor, la palabra sujeto entraña dos significados: uno en el sentido de sujeción o control ejercido por parte de otro; y el segundo, conciencia de sí mismo enlazado a una identidad, que a su vez proviene o es elaborada desde los discursos del poder. En estos dos sentidos se sugieren formas de sometimiento en oposición a la concepción tradicional del sujeto autónomo racional universal (Foucault, 1988).

Foucault también define cuerpos dóciles o el cuerpo político como aquel en el que se imprimen las relaciones de poder, que lo marcan y le exigen signos específicos como respuesta. ${ }^{3}$ Estas relaciones de sujeción no son evidentes de forma directa, sino que se conforman de estrategias, de tácticas — la llamada microfísica del poder- que no necesariamente requieren ejercer violencia, sino que están tan internalizadas en la subjetividad del dominado que pasan a través de él y se incorporan en la socie- 
dad, todos como víctimas y cómplices, sin necesidad de que medien instituciones ni aparatos estatales (Foucault, 1976).

Aludiendo al concepto de cuerpos dóciles, Sandra Bartky cuestiona cómo el cuerpo femenino tiene libertad de movimiento, pero sus movimientos son más restringidos que los de los hombres; está sujeto de forma permanente y exhaustiva a pautas de comportamiento autorreguladas (Bartky, 1994), pero que, como se propone en este trabajo, pueden ser también controladas de forma externa por la comunicación vía celular. Otras autoras (Archambault, 20II; Johnson, 2013; Jouhki, 2013) encontraron en sus investigaciones diversas formas de control hacia las mujeres a través del uso del teléfono celular. Esto permite elaborar un marco para advertir la condición subordinada de las mujeres y el poder disciplinario ubicuo de las relaciones de género.

Retomando las ideas acerca del poder y del proceso de subjetivación de Foucault, Lasén (2014) señala que el teléfono permite la comunicación entre las parejas reproduciendo relaciones desiguales en las que la protección y la seguridad son deseadas e imaginadas como características masculinas por parte de las mujeres, sin cuestionarse la condición de sumisión a la vida privada, como encargadas del cuidado y la crianza de hijos y de otras labores del ámbito doméstico, que son verificadas sistemáticamente a través del celular. Y esta comunicación puede ocurrir en formas en las que los integrantes de la pareja no reconocen que controlan o son controlados, porque "control" tiene una connotación negativa (Lasén, 2014: 28-29).

En este mismo sentido, la autora plantea que se crea una simbiosis persona-dispositivo en función de la accesibilidad y la inmediatez que suponen, refiriéndose a cómo el celular y ciertos rituales se toman como parte de procesos de socialización imprescindibles, tales como el de llevar el teléfono a todas partes y estar accesible a los otros; así como mediante lo que llama inscripción, refiriéndose a la capacidad de los dispositivos de guardar registro de las actividades, que daría a su usuario control, pero también a cualquiera que tenga acceso al dispositivo, entre ellos la pareja. Estas prácticas que involucran control en sí mismas no son percibidas de esa forma porque, particularmente en la relación de pareja, el hecho de estar en comunicación constante y ser transparente es considerado como una demostración de interés y de cuidado o protección por parte del otro.

$\mathrm{Al}$ respecto, Jouhki (2013) observa que el uso del teléfono celular puede beneficiar a las mujeres a través del estrechamiento de lazos, pero a la vez posiblemente refuerza los patrones de dominio patriarcal, ya que suele utilizarse para ejercer medidas de control, por ejemplo, confirmar si están en casa. 
Por su parte, Linke (20I4) identificó que las TIC se emplean de modo distinto en las relaciones sociales de familia en función de la posición, edad y género de cada integrante, y que si las relaciones íntimas de parejas heterosexuales están cada vez menos condicionadas por pautas tradicionales puede existir una mayor negociación y establecimiento de referencias mutuas de comunicación cotidiana más equilibrada en los procesos de construcción de identidad de pareja.

De esta manera, una brecha digital de género se refleja también en la forma distinta en que las TIC son usadas por hombres y mujeres: para el reforzamiento o la persistencia de la actuación en esferas separadas por género; para ejercer supervisión y control sobre el/la otro/otra a través de un teléfono; en la forma como se accede: si se tienen los conocimientos, habilidades, tiempo y libertad para una plena utilización del dispositivo; si se tiene la posibilidad de sufragar su costo; o en la posibilidad de conocer y dominar internet en accesos móviles o fijos, con la finalidad de obtener autonomía en la toma de decisiones individuales, familiares y comunitarias.

Desde este punto de partida, el presente trabajo aspira a complementar, desde una aproximación cuantitativa exploratoria, la visión de las relaciones de género mediadas por el uso de la tecnología celular que en su momento Lasén (20I4) hiciera desde un enfoque cualitativo.

\section{Método y resultados}

Abordar el estudio de las desigualdades de género en el acceso y uso de las TiC requiere revisar y adaptar metodologías utilizadas en otros estudios y a la vez delinear un camino propio para desagregar un fenómeno poco tratado por los estudios de la temática en México (Alva de la Selva, 2015; Mariscal, 2009; Ruelas, 20ı0; 2014; Tello, 2007): la brecha de género y la telefonía móvil.

Averiguar esos usos es relevante para advertir cómo influyen las subjetividades y prácticas de género en la incorporación de estas herramientas tecnológicas a la vida cotidiana, y evaluar con ello las potencialidades que pueden entrañar para resolver, reforzar o redefinir las estructuras de género que mantienen en desventaja a las mujeres, al delimitarles espacios de actuación distintos a los de los hombres en los ámbitos público y privado, asignando culturalmente a las mujeres la responsabilidad central de los trabajos familiares de cuidado y domésticos. 


\section{Acerca de los datos}

En este trabajo se analizan datos de la encuesta Los usos del teléfono celular en Hermosillo realizada en 2016, aplicada a personas adultas en una muestra aleatoria de viviendas del ámbito urbano de la ciudad de Hermosillo, ${ }^{4}$ Sonora, localidad que según la ENDUTiH es la que cuenta con mayor conectividad en el país, donde poco menos de 90\% de los hogares tienen conexión a internet y alrededor de $93 \%$ de la población adulta es usuaria de telefonía celular (INEGI, 20I7; 20I8).

De acuerdo con los datos proporcionados por la Encuesta Intercensal 2015 de INEGI, se estima que en 2016 había 24I 456 viviendas habitadas en la ciudad de Hermosillo. De estas, en 143418 (59.4\%) habitaban hogares encabezados por parejas heterosexuales. En $68.8 \%$ de estas parejas, la edad de las mujeres es menor que la de los hombres (en promedio 5.I años menor); en $12.7 \%$, tienen la misma edad, en tanto que en $18.4 \%$ las mujeres tienen más edad que los hombres (3.5 años en promedio).

Para definir la muestra de viviendas se estratificó la ciudad por sectores socioeconómicos y se eligieron aleatoriamente los hogares a visitar mediante un diseño en tres etapas: en la primera se seleccionaron al azar dos $\mathrm{AGEB}^{5}$ de cada estrato; en la segunda se eligieron aleatoriamente dos manzanas de cada AGEB de la primera etapa; $y$, finalmente, se escogieron al azar (en campo) dos viviendas en cada manzana de la segunda etapa.

El cuestionario, consistente en 75 preguntas, se aplicó entre marzo y mayo de 2016 a una persona adulta habitante de la vivienda que estuviera disponible para responder la encuesta y que en la fase inicial de la entrevista se autodefiniera como usuaria de alguna de las TIC enlistadas en el cuestionario, con el objetivo de identificar las herramientas de acceso más común en la ciudad, los usos regulares y esporádicos, y en particular algunas cuestiones que se relacionen con la división de tareas en la familia.

4 Un procesamiento directo de la base de datos de la Encuesta Intercensal 2015 de INEGI arroja que en ese año la población de la ciudad de Hermosillo ascendía a 819 999 habitantes, representando $28.8 \%$ de la población total de Sonora y $37.4 \%$ de la población asentada en localidades urbanas (de 15000 y más habitantes) de esa entidad. De la población de la ciudad, 51 \% son mujeres y 49 $\%$ son hombres.

5 Área geoestadística básica: espacio geográfico conformado por un conjunto de manzanas, que generalmente va de uno a 50, delimitadas por calles, avenidas, andadores o cualquier otro rasgo de fácil identificación en el terreno y cuyo uso del suelo sea principalmente habitacional, industrial, comercial o de servicios, entre otros. Se puede consultar en <www.inegi.org.mx $>$. 
La información recogida que se utiliza en este trabajo corresponde a 53 personas en edades entre los 20 y los 65 años que encabezan sus respectivos hogares, viven en pareja y son usuarias de la telefonía celular y de las cuales 33 son mujeres y 20 son hombres. El interés sobre este grupo tiene que ver con dos circunstancias: la primera se refiere al interés de averiguar y contrastar los usos de las Tic en general, y los del teléfono celular en particular, que hombres y mujeres desarrollan en las dinámicas de pareja y asociadas con las labores de cuidado familiar; la segunda, relacionada con el grupo de edad, pretende incorporar los comportamientos tanto de personas nativas como no nativas digitales (esto es, personas expuestas a las TIC por primera vez en edad adulta). Por su reducido tamaño, los resultados del análisis de esta muestra deben considerarse de carácter exploratorio. El cuadro I resume las principales características sociodemográficas de las personas que conforman la fuente de datos analizada.

\section{Cuadro i}

Características de las personas encuestadas

\begin{tabular}{lccc}
\hline & Mujeres & Hombres & Total \\
\hline Encuestadas/os & 33 & 20 & 53 \\
\hline Tiempo de relación de pareja & & & \\
\hline De o hasta 5 años & $9 \%$ & $15 \%$ & $11 \%$ \\
\hline Más de 5 años & $91 \%$ & $85 \%$ & $89 \%$ \\
\hline Grupo de Edad & & & \\
\hline 40 o menos años & $42 \%$ & $30 \%$ & $38 \%$ \\
\hline $4 \mathrm{I}-65$ años & $58 \%$ & $70 \%$ & $62 \%$ \\
\hline Nivel máximo de asistencia escolar ${ }^{\mathrm{I}}$ & & & \\
\hline Básico & $61 \%$ & $45 \%$ & $55 \%$ \\
\hline Post básico & $39 \%$ & $55 \%$ & $45 \%$ \\
\hline Ocupación & & & \\
\hline Ocupado/empleado & $62 \%$ & $80 \%$ & $70 \%$ \\
\hline Hogar & $38 \%$ & $0 \%$ & $17 \%$ \\
\hline Desocupado/jubilado & $0 \%$ & $20 \%$ & $13 \%$ \\
\hline Báco:primara, secudaia & & \\
\hline
\end{tabular}

${ }^{1}$ Básico: primaria, secundaria o preparatoria. Posbásico: técnico o superior

Fuente: Encuesta “Los usos del teléfono celular en Hermosillo", 2016 
La mayoría de las personas encuestadas tienen más de cinco años viviendo en su relación de pareja; los hombres tienen en general más edad y escolaridad que las mujeres; por su parte, seis de cada diez mujeres y ocho de cada diez hombres en muestra desarrollaban alguna actividad laboral para el mercado al momento de la encuesta, en tanto que tres de cada diez mujeres y ningún hombre declararon al hogar como su ámbito principal de ocupación. Así que la muestra refleja las diferencias en la conformación de las parejas heterosexuales - en lo relativo a edad, escolaridad y propensión a dedicarse al trabajo para el mercado- que constituyen expresiones de la desigualdad estructural de género y que implican un acceso disminuido de las mujeres a recursos económicos (Lamas, 2016; Hurtado, 2017; Olivera y Arellano, 2017).

Siguiendo la propuesta de análisis de Lasén (20I4), aquí se realiza una aproximación cuantitativa por medio de las respuestas que mujeres y hombres de esta muestra proporcionaron a preguntas específicas, con el fin de explorar las formas en que acceden a la telefonía celular y los usos que unas y otras le dan a esta herramienta tecnológica para comunicarse, mantener los vínculos, gestionar presencias y ausencias, organizar las tareas domésticas y el cuidado de los hijos, así como para vigilar y controlar a la pareja. La estrategia de análisis consistió en contrastar las respuestas de mujeres y hombres a cada una de las cuestiones buscando detectar diferencias o similitudes. Dado el tamaño pequeño de la muestra, se utilizan pruebas no paramétricas para detectar diferencias estadísticamente significativas, a niveles de IO, 5 y I por ciento.

\section{Acceso y conocimiento de TIC}

En la muestra de personas que encabezan sus hogares, sujetos de este trabajo, resultó que las mujeres acceden en promedio a un número menor de las herramientas tecnológicas investigadas en comparación con sus contrapartes masculinas: las mujeres acceden en promedio a alrededor de seis de las diez TIC, mientras que los varones tienen acceso a cerca de ocho en promedio. El cuadro 2 muestra que las diferencias más significativas se ubican en el acceso a internet $y$ a una computadora, seguidas del acceso a telefonía tradicional, ya sea en casa o en la calle. Los resultados señalan también la inexistencia de diferencias estadísticamente significativas en el acceso a las siguientes TIC: radio, TV digital, TV de paga, correo electrónico y redes sociales como Facebook. 


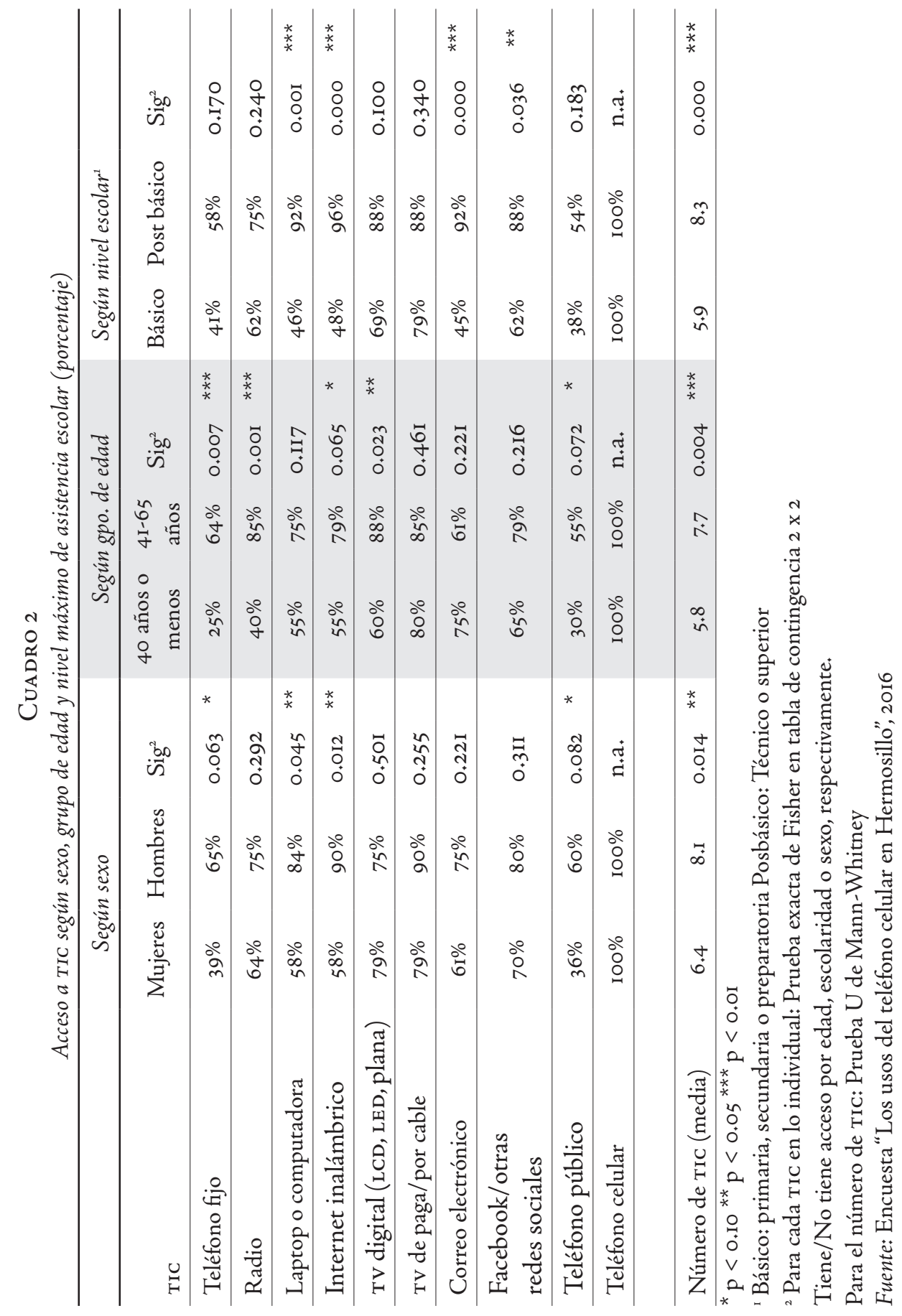


El acceso a Tic es más frecuente en personas mayores de 40 años, tanto en tres de ellas que pueden considerarse tradicionales y que de alguna manera empiezan a ser sustituidas por la telefonía móvil y aplicaciones en internet (radio y telefonía fija o pública), como en el acceso a TV digital e internet inalámbrico, lo que posiblemente esté asociado con diferenciales en el nivel de ingresos y/o distribución del gasto.

Por otra parte, la escolaridad marca la diferencia más amplia en lo relativo al acceso a las Tic, como se observa en las últimas columnas del cuadro 2. En las TiC más recientes - computadora fija o móvil, internet inalámbrico, correo electrónico y redes sociales - se observa un acceso significativamente mayor entre aquellos que han atendido estudios de nivel técnico o superior, comparado con quienes han completado solo los niveles escolares básicos. En cambio, las Tic de mayor antigüedad como radio, telefonía fija (en casa o pública) y televisión arrojan datos similares en ambos grupos.

Además del acceso, el cuestionario indagó si se sabía o no usar cada una de las Tic, mostrándose la inexistencia de diferencias significativas entre hombres y mujeres. Igualmente se revelan como no significativas las diferencias entre las personas en los dos grupos de edad considerados, salvo en el caso del correo electrónico, donde las personas más jóvenes declaran saberlo usar con mayor frecuencia que las personas de 40 y más años. El grado de escolaridad alcanzada sí parece suscitar diferencias significativas - en favor de quienes han realizado estudios más allá de los niveles básicos - en el conocimiento de estas tecnologías (promediando 9.8 herramientas que declaran saber usar quienes tienen escolaridad posbásica contra 8.5 de quienes solo han asistido a educación básica), y particularmente en las siguientes: computadora, internet inalámbrico, correo electrónico, redes sociales. ${ }^{6}$

\section{Acceso al teléfono celular}

Según los resultados de la encuesta, mujeres y hombres declaran tener preferencia por el uso del teléfono celular como medio de información y comunicación. ${ }^{7}$ Casi

6 El cuadro correspondiente no se muestra por razones de espacio, pero está a disposición de quien lo solicite.

7 El teléfono celular es la Tic de acceso más frecuente en los grupos analizados junto con la televisión y las redes sociales. Este resultado está en correspondencia con los datos de INEGI (2017), según los cuales en 2016 Sonora era la entidad con mayor acceso a celulares en el país, con una proporción de usuarios de $87.4 \%$ de la población. En la escala nacional, el IFT reporta que actualmente 9I de cada Ioo habitantes cuentan con una línea móvil (IFT, 20I7: I8). 
el total de personas encuestadas cuentan con un dispositivo móvil propio, ${ }^{8}$ y también prácticamente la totalidad, sin diferencias por sexo, consideraron importante contar con un teléfono móvil propio. ${ }^{9}$ La diferencia estriba en los motivos por los cuales lo consideran así.

\section{Cuadro 3}

Motivos importantes para tener móvil propio

\begin{tabular}{|c|c|c|c|c|}
\hline \multirow[b]{2}{*}{ Motivo } & \multicolumn{4}{|c|}{ Porcentaje de mención } \\
\hline & Mujeres & Hombres & $\mathrm{Sig}^{1}$ & \\
\hline Mantener contacto con familia & $67 \%$ & $60 \%$ & 0.426 & \\
\hline Cualquier contacto le puede localizar & $27 \%$ & $35 \%$ & 0.374 & \\
\hline Mantener contacto con trabajo & $13 \%$ & $55 \%$ & 0.002 & *** \\
\hline Puede localizar a otras personas & $33 \%$ & $20 \%$ & 0.242 & \\
\hline Para emergencias, por seguridad & $20 \%$ & $10 \%$ & 0.297 & \\
\hline Siente angustia cuando no lo tiene & $13 \%$ & $15 \%$ & 0.590 & \\
\hline Obtener información en cualquier momento & $13 \%$ & $5 \%$ & 0.326 & \\
\hline Se siente independiente & $13 \%$ & $5 \%$ & 0.326 & \\
\hline Guardar información valiosa/privada & $7 \%$ & $10 \%$ & 0.528 & \\
\hline
\end{tabular}

${ }^{1}$ Prueba exacta de Fisher en tabla de contingencia 2 × 2 (Mencionado/No mencionado por Sexo Mujeres/Hombres)

Fuente: Encuesta "Los usos del teléfono celular en Hermosillo", 2016

La principal motivación declarada por hombres y mujeres — sin diferencias significativas - para tener celular propio radica en la posibilidad de mantener contacto con la familia. Sin embargo, para los hombres, el celular es prácticamente igual de importante para conectarse con el ámbito del trabajo extradoméstico, en tanto que

8 Solo dos de las 53 personas encuestadas (ambas mujeres) declararon que no era propio el celular que utilizan y casi una cuarta parte - sin diferencias por sexo-que el dispositivo no cuenta con las características de un teléfono inteligente o smartphone, lo que es una limitante para poder beneficiarse del acceso a internet; además de que podría ser subutilizado, pues $80 \%$ reconoció no conocer la mayoría de las funciones que tiene su propio teléfono, lo que puede indicar que las herramientas de aprendizaje para la utilización de nuevas tecnologías no llegan a todos los estratos de la población con la misma rapidez con la que se comercializan. Esto implica que la brecha no se remediaría solo con equipamiento o acceso universal, sino que se requeriría atender otros factores acerca del uso y la posible utilidad que pueden significar estos dispositivos.

9 Solo una persona (varón) declaró que no lo consideraba importante. 
esta motivación es declarada por pocas mujeres. El segundo orden de motivos para hombres y mujeres es el de poder localizar a ser localizado por cualquier otra persona de sus contactos. Atender emergencias o sentir seguridad, incluso al grado de sentir angustia si no se cuenta con el dispositivo, aparece como un tercer orden de importancia. Además, la posibilidad de obtener o guardar información privada en el dispositivo móvil aparece como un motivo muy poco mencionado por hombres y mujeres, al igual que la obtención de un sentimiento de independencia (cuadro 3).

El acceso a teléfono móvil propio no ocurre de igual forma para mujeres que para hombres. Como se aprecia en las figuras i y 2, aproximadamente la mitad de las mujeres recibieron su dispositivo de otra persona, principalmente su pareja, y es esta quien se hace cargo de su costo; en contraste, prácticamente todos los hombres declaran adquirir por sí mismos su dispositivo y pagar su consumo (la mitad coincide en declarar que paga los de toda la familia, incluidos los de la pareja, y la otra mitad los comparte con la pareja o cada quien paga el propio).

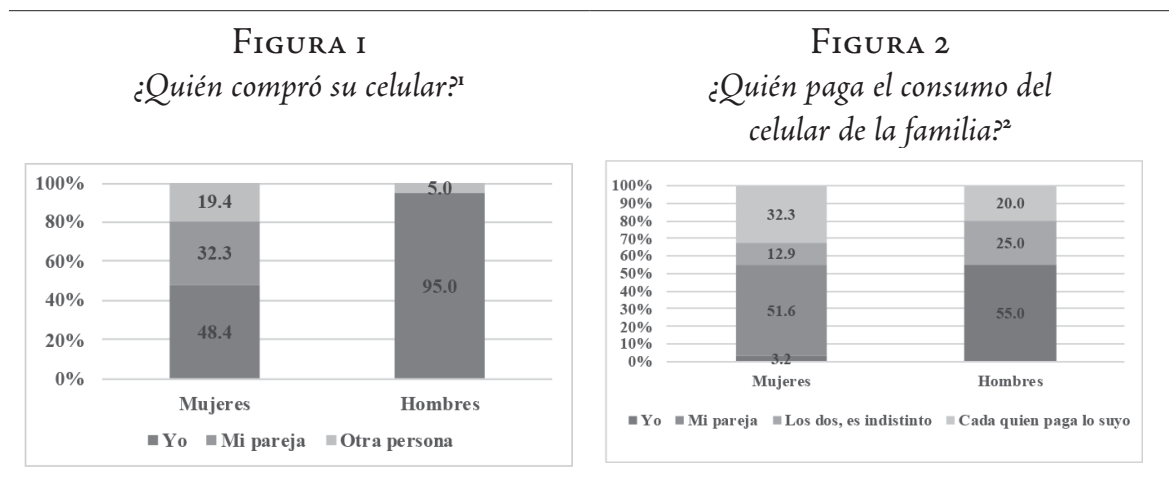

${ }^{1}$ Sig. $0.000^{* * *}$ en prueba exacta de Fisher para variable dicotomizada (Yo/Otros) por sexo

${ }^{2}$ Sig. $0.000^{* * *}$ en prueba chi-cuadrada de homogeneidad

${ }^{*} \mathrm{p}<0.10^{* *} \mathrm{p}<0.05^{* * *} \mathrm{p}<0.01$

Fuente: Encuesta “Los usos del teléfono celular en Hermosillo", 2016

\section{Usos del teléfono celular y la vida en pareja}

Cuestionados acerca de quién les apoyó en el aprendizaje para la utilización de su dispositivo, la mayoría se declaran autosuficientes, en tanto que otros reconocen haberlo hecho con la guía de familiares, principalmente cónyuges, hijos o hijas, con diferencias no estadísticamente significativas entre hombres y mujeres (figura 3 ). Por el contrario, resulta altamente significativa la diferencia con que hombres y mujeres 
se pronuncian acerca del ámbito en el que les es más necesario o prefieren usar su celular (figura 4): mientras que dos de cada tres mujeres señalan su casa, tres de cada cuatro hombres señalan otro lugar o que es cualquier parte.

Figura 3

¿Quién le enseñó a usar su celular?

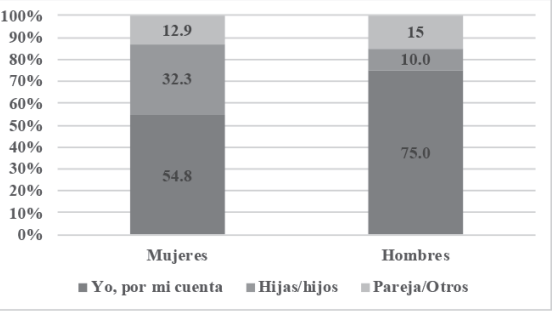

Figura 4

Lugar de mayor necesidad o preferencia para usar el celular?

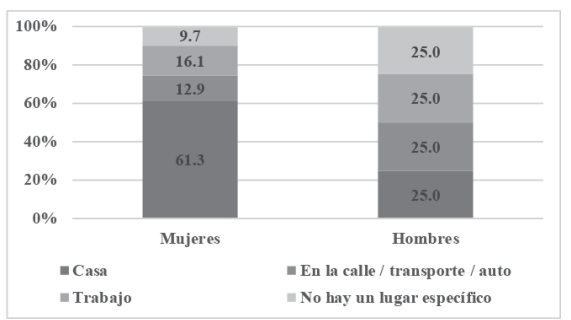

${ }^{\mathrm{I}}$ Sig. 0.123 en prueba exacta de Fisher para variable dicotomizada (Yo, por mi cuenta) Otros) por sexo

${ }^{2} \mathrm{Sig}$. $0.012^{* *}$ en prueba exacta de Fisher para variable dicotomizada (Casa/Otros, no hay lugar específico) por sexo

${ }^{*} \mathrm{p}<0.10{ }^{* *} \mathrm{p}<0.05^{* * *} \mathrm{p}<0.01$

Fuente: Encuesta “Los usos del teléfono celular en Hermosillo", 2016

Los usos más frecuentes del celular fueron consultar la hora o fecha y hacer o responder llamadas, sin distinción entre hombres y mujeres. En el mismo orden de alta frecuencia (varias veces al día) se ubica el uso de mensajería instantánea WhatsApp en el caso de las mujeres, pero no así en el de los hombres, quienes en promedio declararon una frecuencia de uso mucho más baja de esa herramienta de comunicación. En un segundo orden de frecuencia, también sin diferencias entre hombres y mujeres, se encuentra la interacción en redes sociales como Facebook y Twitter (tanto en su faceta social como noticiosa) y a través de mensajes SMs, así como la utilización de la cámara para tomar fotos y video, y navegar en internet. En este grupo destacan las aplicaciones de calendario y agenda, que es utilizada con significativa mayor frecuencia por las mujeres que por los hombres. Por último, descargar contenidos de internet (música, videos, libros, aplicaciones) y el correo electrónico aparecen con las frecuencias de uso promedio más bajas tanto entre las mujeres como entre los hombres (cuadro 4). 


\section{Cuadro 4}

Frecuencia de distintos usos del teléfono celular según sexo

\begin{tabular}{|c|c|c|c|c|}
\hline \multirow[b]{2}{*}{ Uso del teléfono celular } & \multicolumn{3}{|c|}{ Frecuencia ${ }^{1}$ (media) } & \\
\hline & Mujeres & Hombres & $\mathrm{Sig}^{2}$ & \\
\hline Consultar la hora/fecha & 4.8 & 4.6 & 0.787 & \\
\hline Hacer llamadas & 4.6 & 4.7 & 0.663 & \\
\hline Responder llamadas & 4.7 & $4+3$ & 0.353 & \\
\hline WhatsApp/ mensajería instantánea & 4.6 & $3+3$ & 0.006 & *** \\
\hline $\begin{array}{l}\text { Facebook/Twitter: revisar fotos, estados, compartir, } \\
\text { comentar }\end{array}$ & $3+1$ & 2.8 & 0.415 & \\
\hline Mensajes SMs (leer y responder) & 2.9 & 2.9 & 0.890 & \\
\hline Facebook/Twitter: noticias, entretenimiento & 3.0 & 2.5 & 0.311 & \\
\hline Cámara: tomar fotos y video & $3+1$ & 2.2 & 0.133 & \\
\hline Navegar en Internet, hacer consultas & 2.8 & 2.7 & 0.822 & \\
\hline Calendario y agenda de citas & 3.4 & 1.5 & 0.006 & $* * *$ \\
\hline $\begin{array}{l}\text { Descargar contenidos de Internet: música, videos, } \\
\text { libros }\end{array}$ & I.6 & I. 6 & 0.897 & \\
\hline Correo electrónico, leer, enviar. & $\mathrm{I} .6$ & $\mathrm{I} .5$ & 0.786 & \\
\hline Descarga/uso de aplicaciones (apps) & 1.5 & I.6 & 0.636 & \\
\hline
\end{tabular}

${ }^{1}$ La frecuencia de uso se capturó así: $0=$ Nunca, $1=$ Escasamente (menos de 1 vez al mes), 2 $=$ Poco (1-2 veces al mes), 3 = Frecuencia media (1-3 veces por semana), $4=$ Muy frecuente $(1$ vez al día), 5 = Varias veces al día.

${ }^{2}$ En prueba U de Mann-Whitney

${ }^{*} \mathrm{p}<0.10{ }^{* *} \mathrm{p}<0.05^{* * *} \mathrm{p}<0.01$

Fuente: Encuesta "Los usos del teléfono celular en Hermosillo", 2016

En el cuadro 5 se resumen las respuestas a la pregunta sobre la importancia otorgada al teléfono móvil para mantener el contacto con diferentes personas e instituciones. Para hombres y mujeres, el vínculo más apreciado es con el primer núcleo familiar (pareja, hijas e hijos); solo en el caso de las mujeres resulta igualmente importante mantener contacto con el círculo familiar más amplio. El resto de los contactos recibe una valoración inferior y sin distingo, a excepción del contacto con el gobierno, los servicios de emergencia y los servicios municipales, que reciben una valoración más alta por parte de las mujeres que de los hombres. 


\section{Cuadro 5}

Importancia del teléfono celular para mantener contacto según sexo

\begin{tabular}{|c|c|c|c|c|}
\hline \multirow[b]{2}{*}{ Contacto con... } & \multicolumn{4}{|c|}{ Importancia $^{\mathrm{I}}$ (media) } \\
\hline & Mujeres & Hombres & $\mathrm{Sig}^{2}$ & \\
\hline Pareja & 2.8 & 2.6 & 0.425 & \\
\hline Hijos & 2.4 & 2.2 & 0.390 & \\
\hline Otros familiares (padres, hermanos, tíos) & 2.5 & I.8 & 0.022 & ** \\
\hline Trabajo (jefes) & $\mathrm{I}+4$ & I.9 & 0.243 & \\
\hline Amistades/Grupos & I. 5 & $\mathrm{I} .8$ & $0.28 \mathrm{I}$ & \\
\hline Trabajo (compañeros) & I.2 & $\mathrm{I}+7$ & 0.330 & \\
\hline Escuela/educación & I.I & $\mathrm{I}+4$ & 0.568 & \\
\hline Otros servicios para el hogar & 0.9 & $\mathrm{I}+2$ & 0.329 & \\
\hline Salud/médicos/remedios caseros & 0.9 & $\mathrm{I}+\mathrm{O}$ & 0.884 & \\
\hline Gobierno, o66, servicios municipales & I.O & 0.5 & 0.073 & * \\
\hline
\end{tabular}

${ }^{1}$ El grado de importancia se capturó así $\div$ o = Nada importante, $\mathrm{I}=$ Poco importante, 2 = Importancia media, 3 = Muy importante

${ }^{2}$ En prueba U de Mann-Whitney

${ }^{*} \mathrm{p}<0.10{ }^{* *} \mathrm{p}<0.05^{* * *} \mathrm{p}<0.01$

Fuente: Encuesta "Los usos del teléfono celular en Hermosillo", 2016

Prácticamente todas las personas encuestadas declararon usar el celular para contactar a su pareja ${ }^{10}$ varias veces o al menos una vez al día. La mitad señaló utilizarlo para comunicarse mediante llamadas y mensajería; la otra mitad sólo para llamadas. ${ }^{11}$ Aunque hombres y mujeres difieren significativamente en su declaración acerca de la frecuencia con que abordan diversos temas con sus parejas, ambos coinciden en que los asuntos más tratados se relacionan con la familia y los hijos, el trabajo y la economía familiar; en un siguiente nivel se encontrarían temas relacionados con asuntos domésticos, la propia relación de pareja, la salud, las actividades escolares y la familia extendida (véase cuadro 6).

10 Solo un hombre declaró no hacerlo.

11 Solo una mujer declaró usar únicamente mensajería instantánea para comunicarse por celular con su pareja. 


\section{Cuadro 6}

Frecuencia de tratamiento de temas entre la pareja mediante el celular

\begin{tabular}{|c|c|c|c|c|}
\hline \multirow[b]{2}{*}{ Tema } & \multicolumn{2}{|c|}{ Frecuencia $^{1}$ (media) } & \multirow[b]{2}{*}{$\mathrm{Sig}^{2}$} & \\
\hline & Mujeres & Hombres & & \\
\hline ¿Con qué frecuencia habla con su pareja? & 4.3 & 4.2 & 0.949 & \\
\hline Relación familiar/ hijos & $4+2$ & $3+3$ & 0.013 & ** \\
\hline Trabajo y economía familiar & 4.0 & 2.8 & 0.012 & ** \\
\hline $\begin{array}{l}\text { Asuntos domésticos (trámites, reparaciones, servicios, } \\
\text { seguridad, etc.) }\end{array}$ & $3+5$ & 2.7 & 0.072 & * \\
\hline Relación de pareja/ vida íntima & 3.4 & 2.8 & 0.316 & \\
\hline Salud & 3.6 & 2.4 & 0.023 & ** \\
\hline Educación/asuntos escolares & $3+3$ & 2.1 & 0.030 & ** \\
\hline $\begin{array}{l}\text { Familia extendida (padres, hermanos, primos, familia } \\
\text { politica, etc) }\end{array}$ & 3.1 & 2.0 & 0.074 & * \\
\hline Amistades/Grupos & 2.7 & 2.5 & 0.744 & \\
\hline $\begin{array}{l}\text { Servicios públicos (agua, drenaje, electricidad, pavi- } \\
\text { mentación, alumbrado, seguridad, etc.) }\end{array}$ & 2.5 & 2.2 & 0.522 & \\
\hline $\begin{array}{l}\text { La frecuencia se capturó así: } 0=\text { Nunca, } 1 \text { = Escasame } \\
(1-2 \text { veces al mes), } 3=\text { Frecuencia media ( } 1-3 \text { veces por } \\
\text { dia), } 5=\text { Varias veces al día. } \\
\text { En prueba U de Mann-Whitney } \\
{ }^{\mathrm{p}}<0 . \mathrm{IO}^{* *} \mathrm{p}<0.05^{* * *} \mathrm{p}<0.0 \mathrm{I}\end{array}$ & $\begin{array}{l}\text { nte (menos } \\
\text { mana), } 4=\end{array}$ & $\begin{array}{l}\text { de } 1 \text { vez al } m \\
\text { Muy frecu }\end{array}$ & $\begin{array}{l}\text { es) }, 2= \\
\text { nte }(1 v\end{array}$ & \\
\hline
\end{tabular}

Cuando se pregunta acerca de los usos que dan al celular en el entorno familiar, las mujeres declaran ser principalmente ellas quienes reciben las solicitudes de ayuda, mientras que solo la mitad de los varones declara que les llaman a ellos (véase figura 5). La diferencia de respuestas es estadísticamente significativa.

Con el fin de identificar señales de dominio o control mediado por el teléfono, en el cuestionario se incluyeron 15 reactivos, ${ }^{12}$ con los cuales se construyó un indicador resumen. Las preguntas exploraron desde aspectos del uso del teléfono (consumo, contactos, llamadas, mensajes, publicaciones en redes sociales, entre otros) en los que no se tiene libertad de decisión y/o deben consultarse; si el celular propio es usado por la pareja sin consentimiento; o la declaración misma de tener impedimen-

12 Las preguntas pueden consultarse en el cuadro 7. 


\section{Cuadro 7}

Preguntas incluidas en el indicador de control ${ }^{*}$

\begin{tabular}{|c|c|c|c|}
\hline Número & Pregunta & \multicolumn{2}{|c|}{ Respuesta/Codificación } \\
\hline 33 & $\begin{array}{l}\text { ¿Hay algún impedimento de su pareja hacia } \\
\text { usted para su pleno uso del celular? }\end{array}$ & o No & I Sí \\
\hline 38 & $\begin{array}{l}\text { Señale aspectos en los que Ud. no tiene libertad } \\
\text { de decisión en su propio teléfono celular o que } \\
\text { tiene que consultar con su pareja }\end{array}$ & $\begin{array}{l}\text { o No men- } \\
\text { cionado }\end{array}$ & $\begin{array}{l}\text { I Mencio- } \\
\text { nado }\end{array}$ \\
\hline 38_I & $\begin{array}{l}\text { I Tiempo aire que consume/ monto de su } \\
\text { factura }\end{array}$ & “ & “ \\
\hline $38 \_2$ & 2 Sus contactos & “ & “ \\
\hline $38 \_3$ & 3 Tiempo u horarios de uso del teléfono & “ & “ \\
\hline 38_4 & 4 Número de llamadas & “ & “ \\
\hline $38 \_5$ & 5 Duración de llamadas & “ & “ \\
\hline $38 \_6$ & 6 Contenidos de internet que mira o busca & “ & “ \\
\hline 38_7 & 7 Fotos que mira o toma & “ & “ \\
\hline $38 \_8$ & 8 Mensajes de texto/Whatsapp & “ & “ \\
\hline 38_9 & 9 Publicaciones que hace en redes sociales & “ & “ \\
\hline 38_10 & Io Otros & “ & “ \\
\hline 46 & $\begin{array}{l}\text { ¿Su pareja usa o busca información en el telé- } \\
\text { fono de Usted? }\end{array}$ & o No/No sé & $\begin{array}{l}\text { I Sí, siem- } \\
\text { pre/A veces }\end{array}$ \\
\hline 49 & ¿Su pareja tiene permiso para hacerlo? & o Sí, siempre & $\begin{array}{l}\text { I A veces/ } \\
\text { No }\end{array}$ \\
\hline 58 & $\begin{array}{l}\text { ¿Siente usted autonomía o libertad para usar } \\
\text { su celular? }\end{array}$ & o Sí, siempre & $\begin{array}{l}\text { I A veces/ } \\
\text { No }\end{array}$ \\
\hline 60 & $\begin{array}{l}\text { ¿Alguna vez siente que es supervisado/contro- } \\
\text { lado por su pareja a través del teléfono? }\end{array}$ & ○ No & $\begin{array}{l}\text { I Sí, siem- } \\
\text { pre/A veces }\end{array}$ \\
\hline
\end{tabular}

* El indicador "Siente/experimenta control" de la pareja a través del celular se definió como :
$1(\mathrm{Si})$ : Si alguna de las respuestas fue 1 (menos la 46 y 49, que ambas debian ser 1)
$o(\mathrm{No} / \mathrm{NE})$ : De otra manera

Fuente: Encuesta “Los usos del teléfono celular en Hermosillo", 2016 
tos para el uso pleno de su dispositivo por parte de la pareja o sentir supervisión/ control a través del teléfono.

De acuerdo con los resultados, alrededor de una tercera parte de quienes participaron en esta encuesta declaran alguna experiencia o sentir control por parte de la pareja a través del uso del celular, con diferencias por sexo que no alcanzan a distinguirse como significativas, como se aprecia en la figura 6 .

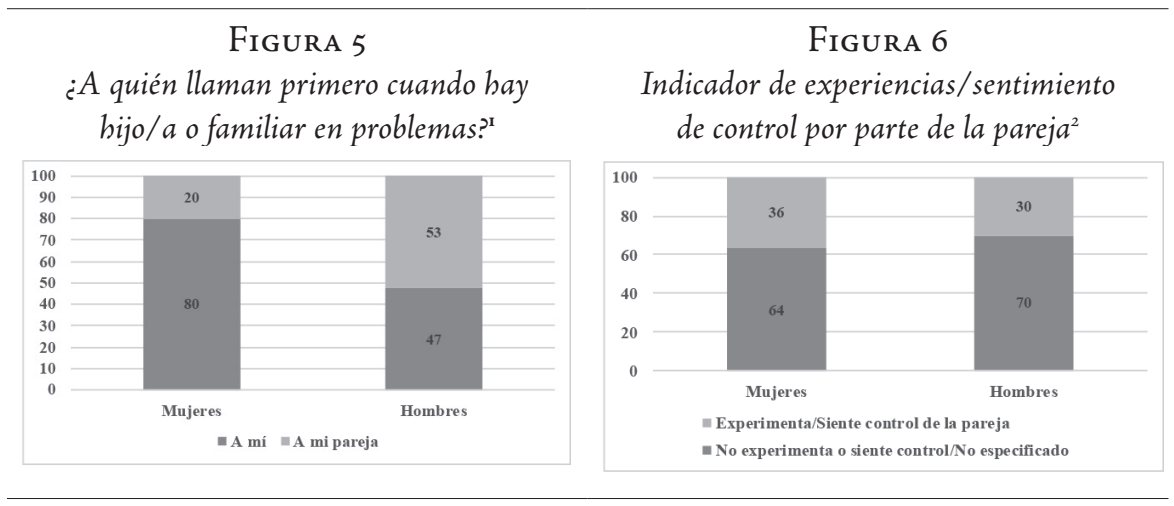

${ }^{1}$ Sig. $0.020^{* *}$ en prueba exacta de Fisher ${ }^{*} \mathrm{p}<0.10^{* *} \mathrm{p}<0.05^{* * *} \mathrm{p}<0.01$

${ }^{2}$ Sig. 0.434 en prueba exacta de Fisher ${ }^{*} p<0.10{ }^{* *} p<0.05^{* * *} p<0.01$

Fuente: Encuesta “Los usos del teléfono celular en Hermosillo", 2016

\section{Discusión}

La mayoría de las mujeres en sus declaraciones asumieron la comunicación con la familia y al ámbito de la casa como el motivo y el espacio que explican su necesidad o preferencia por utilizar el móvil, en tanto que los hombres tienen presentes todos sus ámbitos de actuación. También se observó que en muchos casos el acceso de las mujeres al teléfono móvil es a través de sus parejas, que puede no ser únicamente una cuestión de falta de recursos propios, sino también del reconocimiento de la relación de pareja, que subjetivamente involucra dependencia y control, como también se encontró en otros trabajos (Lasén, 2014; Johnson, 2013; Jouhki, 2013).

Pese al menor porcentaje de acceso a computadora e internet fijo, las mujeres logran a través de un teléfono celular el acceso a redes sociales como Facebook y declaran un mayor uso de WhatsApp que los hombres. Esto les permitiría obtener dominio de herramientas TIC y una presencia en el espacio virtual que disminuiría 
el aislamiento y ampliaría sus espacios de comunicación al constituirse en parte de una comunidad, lo que puede favorecer ciertas formas de empoderamiento femenino mediante la obtención de reconocimiento, en este caso a través de una página o la pertenencia a grupos en estas redes sociales, en concordancia con Huyer y Hafkin (2006) y Parpart, Rai y Staudt (2002).

Contar con las herramientas virtuales supone para hombres y mujeres que hay un flujo de comunicación desde y hacia la familia nuclear, y en particular que la comunicación virtual por medio de llamadas y mensajes entre la pareja es uno de los usos más frecuentes del teléfono móvil. Por su conducto se establecen vínculos que les permiten desempeñar su papel de madres y padres, tal y como sucede al hablar o intercambiar mensajes para tratar asuntos familiares, de trabajo o económicos, compartir fotos o videos de los hijos a través de una red social, así como relacionarse como pareja a la distancia tratando en esta virtualidad asuntos de su vida íntima y su propia relación.

En el caso de las mujeres, se encuentran más elementos de la utilización del celular para gestionar sus roles familiares y extradomésticos, como su declaración de vincularse más estrechamente que los hombres no solo con su familia nuclear sino con la ampliada, o ser a quien más se recurre por este medio en caso de cualquier necesidad o emergencia de otros integrantes de la familia, o utilizar más que los hombres la agenda del celular para coordinar sus múltiples tareas y las páginas web como las de e-gobierno para estar al pendiente de los trámites, pagos y estado de los servicios municipales. Esto indicaría, por una parte, que las mujeres siguen ejerciendo el papel de cuidadora aun a distancia, resolviendo situaciones para otros mediante el celular. En el caso de las informantes que trabajan fuera del hogar, las responsabilidades que desempeñan sobre los asuntos domésticos no decrecen por la existencia de las TIC, de modo que podría percibirse que tienen mayores necesidades de conexión y comunicación para ser localizadas (o estar disponibles) a través de distintos medios.

Por lo que respecta a la respuesta de los varones de otorgar la mayor importancia de tener un celular a su comunicación con la pareja, hijas e hijos también puede expresar que están participando o están dispuestos a tener mayor participación en el cuidado y la atención hacia otros miembros de la familia; es decir, que las jerarquías e identidades de género se pueden renegociar a partir de la presencia de dispositivos tecnológicos que incentivan el contacto constante.

Las posibilidades de accesibilidad, contacto permanente y presencia virtual de la telefonía celular potencian la comunicación entre las parejas, así como las capacidades de supervisión y control en lo que Lasén (2014) refiere como una correa 
inalámbrica - y se podría agregar también invisible-: el hábito de estar accesible, hacer y recibir llamadas y mensajes para informar en cualquier momento dónde se está y qué se hace no se considera usualmente vigilancia y control, sino "estar conectados" e, incluso, pueden considerarse señales de interés y apego de parte de la pareja.

Lasén (20I4) agrega otras dos características en la utilización de la telefonía celular que resultan elementos de control clave en las relaciones de pareja: la capacidad de inscripción o registro de los dispositivos (de tiempos, contactos, llamadas, mensajes, almacenamiento de fotos y multimedia, conversaciones en redes, páginas de navegación, entre otros) que permiten una mirada autorreflexiva y construir parcelas de identidad para quien lo posee, pero también para cualquiera que tenga acceso al aparato; el otro elemento es la exigencia de transparencia equiparada a confianza que se considera signo de una buena relación, en la que se puede acceder a cualquier nivel del dispositivo si la pareja "no tiene nada que ocultar".

En este estudio se encontraron indicios claros de que una buena parte de hombres y mujeres experimentan limitaciones al uso de su dispositivo celular o sienten franco control por parte de sus parejas a través de este, lo que parece parte de las relaciones de pareja en la actualidad que ameritan ser estudiadas con mayor amplitud y precisión.

\section{Conclusiones}

Desde las primeras etapas de la investigación se tuvo evidencia de que los estudios, reportes y estadísticas generados desde los organismos oficiales muestran un panorama parcial de hábitos entre usuarias y usuarios de TIC, lo que sumado a la poca desagregación de la información ofrece poco margen para definir nuevas vías de acción en la solución de las barreras presentes, que van más allá de la falta de acceso a equipamiento.

El estudio presentado se propuso explorar la relación entre el uso de las TIC y la acentuación de los roles de género a partir de una aproximación cuantitativa que permitiera identificar la forma $e$ intensidad en que personas usuarias adultas no expertas en usos digitales, integrantes de parejas heterosexuales, utilizan el celular. Las evidencias mostradas responden en gran parte al objetivo planteado; se consiguió delinear ciertas condiciones de uso del teléfono celular que muestran signos de inequidad que configuran una brecha entre la posibilidad de acceso a TIC por parte de las mujeres y el beneficio real que puedan obtener de dichas herramientas. 
Las pruebas estadísticas fortalecen la validez de las diferencias encontradas entre el grupo analizado.

Los resultados mostraron una brecha en el acceso a computadora e internet $y$, en menor medida, en el acceso a telefonía fija entre hombres y mujeres. Estos hallazgos son compatibles con las perspectivas que asocian la brecha digital de género, en lo relativo al acceso a las Tic, a limitantes en el acceso a recursos por parte de las mujeres, así como a la mayor probabilidad de los hombres de desenvolverse en un contexto de exposición a la tecnología (Castaño, 2008; Gil-Juarez et al, 20II; Wajcman, 2000).

En Hermosillo, donde una importante proporción de las mujeres (alrededor de $40 \%$ ) pertenece a la población económicamente activa, se esperaba que los hallazgos fueran en el sentido de que los usos de internet y en particular del teléfono celular estuvieran relacionados con las tareas de telecuidado hacia los hijos u otros miembros de la familia y que los usos digitales ocurrieran en términos de desigualdad. Se pueden identificar ciertos rasgos que indican sobrecargas de género en el dato de atención más frecuente a llamadas de los hijos, o las diferencias significativas en la importancia del contacto que mantienen principalmente las mujeres con la familia extendida.

Las distinciones encontradas entre hombres y mujeres en lo relativo a las razones por las cuales se considera importante contar con un teléfono celular, los ámbitos en que declaran tener mayor necesidad o preferencia para su uso, así como las formas en que unos y otras acceden a la telefonía celular y cubren sus costos, aparecen como expresiones consistentes de algunos aspectos de la construcción social de género más tradicional. Por una parte, como manifestación de la presencia naturalizada de los hombres en todos los ámbitos (privado y público), y de las mujeres principalmente en el privado; por otra, de la naturalización del rol de los hombres como proveedores de sus familias y de las mujeres como sujetos con un menor acceso a recursos económicos propios y a la toma de decisiones sobre la adquisición de cierto tipo de bienes. En cualquier caso, las diferencias remiten a los procesos de subjetivación de esta estructura de género en las relaciones de pareja, que demandan el reconocimiento mutuo de sus integrantes en maneras que entrañan el establecimiento de relaciones de sujeción y dependencia como señala Foucault.

La atención a las necesidades de los hijos y de otros miembros de la familia forma parte de la subjetividad de género en las mujeres que participaron en el estudio, de modo que el dispositivo electrónico resulta un medio para continuar ejerciendo prácticas de dominio de género en el sentido que anteriormente encontraron Castells (2007) y Lasén (2014). 
Finalmente, es conveniente subrayar que los datos existentes relativos al uso de tecnologías de la información por parte de las mujeres de todos los sectores socioeconómicos son aún limitados, por lo que este campo de estudio presenta todavía desafíos metodológicos y teóricos por desarrollar.

\section{Referencias bibliográficas}

Alva de la Selva, Alma Rosa

2015 "Los nuevos rostros de la desigualdad en el siglo xxi: la brecha digital", Revista Mexicana de Ciencias Politicas y Sociales, 6o(223), pp. 265-285. https://doi.org/Io.IoI6/Sor85-1918(I5)72138-0.

Archambault, Julie $S$.

201 "Breaking up 'because of the phone' and the transformative potential of information in Southern Mozambique", New Media E Society, 13(3), pp. 444-456. https://doi.org/IO.I177/1461444810393906

Bartky, Sandra L.

I994 "Foucault, feminismo y la modernización del poder patriarcal", en E. Larrauri (ed.), Mujeres, derecho penal y criminología, México, Siglo xxI Editores, pp. 63-92.

Bimber, Bruce

2000 "Measuring the gender Gap on the Internet", Social Science Quarterly, 8I(3), pp. 868-876. Recuperado de <http://www.jstor,org/stable/428640Io>.

Bourdieu, Pierre

2000 La dominación masculina, Barcelona, Anagrama.

Brito, Myriam

2016 "División sexual del trabajo: espacio público, espacio privado, espacio doméstico", en Hortensia Moreno y Eva Alcántara (eds.), Conceptos clave en los estudios de género México, Programa Universitario de Estudios de Género-unam, pp. 63-76.

Castaño, Cecilia

2008 "La segunda brecha digital y las mujeres jóvenes. Mujeres en Red”, El periódico feminista, pp. 218-224. Recuperado de <http://www.mujeresenred.net/spip.php?articleI567>.

Castells, Manuel et al.

2007 Comunicación móvil y sociedad, una perspectiva global, Madrid, Ariel. 
De Barbieri, Teresita

I993 "Sobre la categoría género: una introducción teórico-metodológica”, Debates en Sociología, I8, pp. I45-169. Recuperado de <http:// revistas.pucp.edu.pe/index.php/debatesensociologia/article/ view/6680/6784>.

Foucault, Michel

1976 "Los cuerpos dóciles", en Vigilar y castigar. El nacimiento de la prisión, México, Siglo xxi Editores, pp. I39-I74.

1988 El sujeto y el poder, Revista Mexicana de Sociología, 5o(3), pp. 3-20.

Gil-Juarez, Adriana, Ana Vitores, Joel Feliu y Montse Vall-llovera

201 "Brecha digital de género: una revisión y una propuesta", Teoría de la Educación y Cultura en la Sociedad de la Información, I2(2), pp. 25-53.

Hilbert, Martin

201 "Digital gender divide or technologically empowered women in developing countries? A typical case of lies, damned lies, and statistics", Women's Studies International Forum, 34(6), pp. 479-489. <https:// doi.org/I0.1016/j.wsif.2011.07.001>.

Hurtado, Teodora

2017 "La nueva división internacional del trabajo femenino en el contexto de la crisis socioeconómica contemporánea”, en Flor Marina Bermúdez y Clara Luz Villanueva (coords.) Mujeres ante la crisis económica global: empoderamiento y precariedad, México, Universidad de Ciencias y Artes de Chiapas / Miguel Ángel Porrúa

Huyer, Sofia y Nancy Hafkin

2006 Cinderella or Cyberella? Empowering women in the knowledge society, Estados Unidos, Kumarian.

Instituto Federal de Telecomunicaciones (IFT)

2017 "Primer Informe Trimestral 20I7", Recuperado de http://www.ift.org. $\mathrm{mx} /$ comunicacion-y-medios /informes/informes-estadisticos-rer- trimestre-2017.

Instituto Nacional de Estadística y Geografía (INEGI)

2017 "Encuesta Nacional de Uso de Tecnologías de la Información en Hogares, ENDUTiH 2016", Recuperado de <http://www.inegi.org.mx/saladeprensa/boletines/2017/ especiales/especiales2017_03_02.pdf>. 
2018 "Encuesta Nacional de Uso de Tecnologías de la Información en Hogares, ENDUTIH 20I7". Recuperado de <https://www.inegi.org.mx/contenidos/saladeprensa/boletines/2018/OtrTemEcon/ENDUTIH2OI8_02. pdf $>$.

Johnson, Michelle

2013 "Culture's Calling: Mobile Phones, Gender, and the Making of an African Migrant Village in Lisbon", Anthropological Quarterly, 86(I), Jouhki, Jukka

pp. 163-190. Recuperado de <http://www.jstor.org/stable/41857315>.

2013 "A phone of one's own? Social value, cultural meaning and Gendered use of the mobile phone in South India", Suomen Antropologi: Journal of the Finnish Anthropological Society, 38(I), pp. 37-58.

Kumar, Geeta, Nirupama Prakash y Alka Sharma

2017 "The Role of Mobile Phones in Enabling Changes in Gender Relations", Asian Journal of Research in Social Sciences and Humanities, 7(8), pp. 152-I68, DOI: 10.5958/2249-7315.2017.00413.0.

Lamas, Marta

2016 "Género", en Hortensia Moreno y Eva Alcántara (eds.), Conceptos clave en los estudios de género México, Programa Universitario de Estudios de Género-unam, pp. 155-170.

Lasén, Amparo

2014 "Remediaciones móviles de subjetividades y sujeciones en relaciones de pareja”, en Amparo Lasén y Elena Casado (eds.), Mediaciones tecnológicas: cuerpos, afectos y subjetividades, Madrid, Centro de Investigaciones Sociológicas, pp. 21-37.

Lasén, Amparo y Elena Casado (eds.)

2014 Mediaciones tecnológicas: cuerpos, afectos y subjetividades, Madrid, Centro de Investigaciones Sociológicas.

Linke, Christine

2014 "TIC, movilidad y el cambio en la comunicación diaria dentro de las relaciones de pareja”, en Amparo Lásen y Elena Casado (eds.), Mediaciones tecnológicas: cuerpos, afectos y subjetividades Madrid, Centro de Investigaciones Sociológicas, pp. 39-56)..

Mariscal, Judith

2009 "Mobile opportunities: poverty and mobile telephony in Latin America and the Caribbean. The case of Mexico", en Mireia Fernández-Ardèvol y Adela Ros Híjar (eds.), Communication technologies in Latin Ame- 
rica and Africa: A multidisciplinary perspective, Barcelona, Universitat Oberta de Catalunya, pp. 87-II6.

Olivera, Mercedes y Mauricio Arellano

2017 “Podemos hablar de la feminización de la economía campesina marginal de Chiapas? Aportes a una discusión", en Flor Marina Bermúdez y Clara Luz Villanueva (coords.) Mujeres ante la crisis económica global: empoderamiento y precariedad, México, Universidad de Ciencias y Artes de Chiapas / Miguel Ángel Porrúa.

Parpart, Jane, Shirin Rai y Kathleen Staudt

2002 "Rethinking em(power)ment, gender and development: an introduction", en Jane Parpart y Shirin Rai (eds.), Rethinking empowerment. Gender and development in a global/local world, Londres, Routledge.

Pujol, Joan y Marisol Montenegro

2015 "Technology and Feminism: A Strange Couple", Revista de Estudios Sociales, 5I, pp. 173-185.<https://doi.org/10.7440/res51.2015.13>.

Ruelas, Ana Luz

2014 "El teléfono celular y los jóvenes sinaloenses. Adopción, usos y adaptaciones", Comunicación y Sociedad, 2I, pp. IOI-I3I. https://doi. org/I0.32870/cys.voi21.573.

Ruelas, Ana Luz

2010 "El teléfono celular y las aproximaciones para su estudio", Comunicación y Sociedad, I4, pp. I43-167.

Scott, Joan

I996 "El género: una categoría útil para el análisis histórico", en Marta Lamas (comp.), El género: una construcción cultural de la diferencia sexual, México, pueg-unam / Porrúa, pp. 265-30I.

Servon, Lisa y Randal Pinkett

2006 "Estrechando la brecha digital: potencial y límites del Movimiento de Tecnología Comunitaria Norteamericano", en Manuel Castells (ed.), La

Sociedad Red: una visión global, Madrid, Alianza Editorial, pp. 390-412. Tello, Edgar

2007 "Las tecnologías de la información y comunicaciones (TIC) y la brecha digital: su impacto en la sociedad de México", Universities and Knowledge Society Journal, 4(2), pp. I-8.

UIT/UNESCO

2013 Doubling Digital Opportunities. Enhancing the Inclusion of Women and Girls In the Information Society. A report by the Broadband Commission. 
Recuperado de <https://www.broadbandcommission.org/Documents/publications/bb-doubling-digital-2013.pdf >

Vega, Aimée

2016 "Medios de comunicación y nuevas tecnologías", en Hortensia Moreno y Eva Alcántara (eds.), Conceptos clave en los estudios de género, México, PUEG-UNAM, pp. 215-23I.

Wajcman, Judy

2000 "Reflections on Gender and Technology Studies: in what State is the Art?", Social Studies of Science, 30(3), pp. 447-464, https://doi. org/10.1177/030631200030003005.

Wajcman, Judy y Paul Jones

2012 "Border communication: media sociology and sts", Media, Culture $\mathcal{E}$ Society, 34(6), pp. 673-69o. https://doi.org/ı.II77/o163443712449496.

Ahimee Viridiana González Rodríguez

Doctora en Ciencias Sociales por El Colegio de Sonora, especializada en la línea de investigación en Desarrollo y Exclusión Social. Actualmente se desempeña como docente en la Universidad Estatal de Sonora.

Lorenia Velázquez Contreras

Profesora investigadora de El Colegio de Sonora en el Centro de Estudios del Desarrollo. Doctora en Ciencias Económicas por la UABC, trabaja en la línea de investigación de "Desarrollo, sustentabilidad y género", sobre las siguientes áreas de interés: Sustentabilidad y calidad de vida; Desarrollo económico regional.

Gabriela Grijalva Monteverde

Profesora investigadora de El Colegio de Sonora en el Centro de Estudios del Desarrollo. Doctora en Ciencias Económicas por la UABC, trabaja en la línea de investigación de "Desarrollo, sustentabilidad y género", sobre las siguientes áreas de interés: Género y mercados laborales; Bienestar y Pobreza; Segregación, discriminación y salarios. 
Citar como: Ahimee Viridiana González Rodríguez, et al. (2020),"Viviendo con teléfono y pareja. Usos del celular en entornos de alta conectividad", Iztapalapa. Revista de Ciencias Sociales y Humanidades, núm. 89, año 4I, julio-diciembre de 2020, ISSN: 2007-9176; pp. I5I-I8I. Disponible en <http:// revistaiztapalapa.izt.uam.mx/index.php/izt/issue/archive >. 